\title{
Traumatismo craneoencefálico leve en el departamento de urgencias de pediatría del Hospital de Clínicas de San Lorenzo: características clínico epidemiológicas y frecuencia
}

\section{Mild head injury in the pediatric emergency department of the San Lorenzo Clinical Hospital: clinical-epidemiological characteristics and frequency}

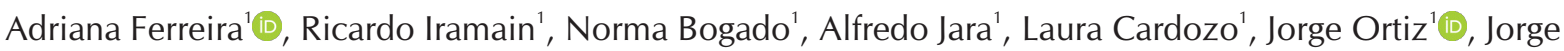

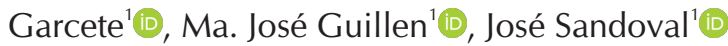 \\ ${ }^{1}$ Universidad Nacional de Asunción. Hospital de Clínicas. Departamento de Urgencias Pediátricas. San \\ Lorenzo, Paraguay.
}

\section{RESUMEN}

Introducción: El traumatismo craneoencefálico ocurre comúnmente en la infancia. La mayoría de los traumatismos craneales en niños son leves y no están asociados con lesiones cerebrales o secuelas a largo plazo. Sin embargo, un pequeño número de niños que parecen estar en bajo riesgo puede tener una lesión cerebral traumática clínicamente importante. Objetivo: determinar la frecuencia, características clínicas y epidemiológicas del traumatismo cráneo encefálico leve en el departamento de emergencias pediátricas del hospital de clínicas de San Lorenzo. Materiales y Métodos: estudio observacional, descriptivo, retrospectivo de corte transversal, se incluyeron pacientes menores a 18 años con diagnóstico de Traumatismo craneoencefálico leve que ingresan a sala de observación del Departamento de Urgencias del Hospital de Clínicas desde noviembre del 2017 hasta noviembre del 2019. Resultados: fueron ingresados 55 pacientes con diagnóstico de TCE leve, el 53\% del sexo masculino, el 36\% pertenecían a lactantes mayores, la mayoría procedían del área metropolitana. En cuanto al mecanismo de traumatismo el $62 \%$ fue por caída de propia altura con un promedio de $0,9 \pm 0,91 \mathrm{~m}$, el $20 \%$ presento pérdida del conocimiento. Todos los pacientes ingresaron al departamento de urgencias vigiles y con un Glasgow 15/15, en cuanto a los hallazgos radiológicos se constató fractura de cráneo en $5 \%$ Se realizo estudios de imagen en el $55 \%$ de

\section{ABSTRAC}

Introduction: Traumatic brain injury occurs commonly in childhood. Most head injuries in children are mild and are not associated with long-term brain injuries or sequelae. However, a small number of children who appear to be at low risk may have a clinically important TBI. Objective: to determine the frequency, clinical and epidemiological characteristics of mild head trauma in the pediatric emergency department of the San Lorenzo Clinical Hospital. Materials and Methods: this was an observational, descriptive, retrospective and crosssectional study that included patients under 18 years of age with a diagnosis of mild head injury who were admitted to the observation room of the Emergency Department of the Clinical Hospital from November 2017 to November 2019. Results: 55 patients with a diagnosis of mild TBI were admitted, $53 \%$ male, $36 \%$ were older infants, the majority came from the metropolitan area. Regarding the trauma mechanism, $62 \%$ was due to a fall from their own height with an average of $0.9 \pm 0.91 \mathrm{~m}, 20 \%$ presented loss of consciousness. All patients were admitted to the emergency department awake and with a Glasgow 15/15, regarding the radiological findings, a skull fracture was confirmed in 5\%. Imaging studies were performed in $55 \%$ of the patients, more than $60 \%$ of these were normal. Conclusion: in patients with mild head injury, doctors must decide whether the patient will

Correspondencia: Adriana Leticia Ferreira Pascottini Correo: adrilferreirap@gmail.com

Recibido: 01/11/2020 Aceptado:12/02/2021

Conflictos de interés: Los autores declaran no presentar conflicto de interés.

DOI: https://doi.org/10.31698/ped.48012021010

\begin{tabular}{|l|l|l}
\hline (cc) BY & Este es un artículo publicado en acceso abierto bajo una Licencia Creative Commons CC-BY 4.0
\end{tabular} 
los pacientes en donde más del $60 \%$ fueron normales. Conclusión: en pacientes con traumatismo craneoencefálico leve los médicos deben decidir si el paciente se realizará una tomografía en base al juicio clínico y a guías internacionalmente estandarizadas para tal efecto ya que las mismas exponen a radiaciones ionizantes que aumentan los riesgos a largo plazo de neoplasias letales. Esto permite que los niños con riesgo bajo a intermedio no sean expuestos innecesariamente a radiaciones.

Palabras claves: traumatismo craneocerebrales, epidemiologias, emergencias tomografía simple, pediatría.

\section{INTRODUCCIÓN}

El traumatismo craneoencefálico ocurre comúnmente en la infancia. La mayoría de los traumatismos craneales en niños son leves y no están asociados con lesiones cerebrales o secuelas a largo plazo. Sin embargo, un pequeño número de niños que parecen estar en bajo riesgo puede tener una lesión cerebral traumática clínicamente importante $(\mathrm{ciTBI})^{(1,2)}$.

El Traumatismo craneoencefálico leve (TCE leve) se define típicamente como leve por un puntaje de la Escala de coma de Glasgow (GCS) de 14 a 15, medido aproximadamente 30 minutos después del tarumatismo $^{(2,3)}$.

El desafío clínico para evaluar un traumatismo craneoencefálico menor en pacientes pediátricos es identificar a los niños con ciTBI al tiempo que limita la imagen radiográfica innecesaria y la exposición a la radiación ${ }^{(3)}$. La neuroimagen, generalmente con tomografía computarizada (TC), es muy sensible para identificar la lesión cerebral que requiere intervención aguda. Sin embargo, los predictores clínicos individuales para ciTBI a menudo son inespecíficos, particularmente en niños pequeños ${ }^{(4,5)}$. Por lo tanto, la evaluación de hallazgos de alto riesgo y el uso de una regla de decisión clínica puede proporcionar un enfoque equilibrado que identifique a casi todos los niños con ciTBI después de un traumatismo craneal menor sin uso excesivo de TC. La mayoría de los lactantes y niños con traumatismos craneoencefálicos leves pueden ser dados de alta de manera segura después de una evaluación cuidadosa sin someterse a imágenes. Si undergo a tomography based on clinical judgment and internationally standardized guidelines for this purpose, since they expose them to ionizing radiation that increases the long-term risks of lethal neoplasms. This allows low to intermediate risk children to not be unnecessarily exposed to radiation.

Key words: Head trauma, epidemiology, simple tomography emergencies, pediatrics.

se realizan neuroimágenes, aquellos pacientes con hallazgos clínicos e imágenes normales también pueden ser dados de alta $^{(6,7)}$.

Al ser una patología común en los niños y a la vez que a pesar de poder ser benigno en la mayoría de los $\operatorname{casos}^{(8)}$ en algunos puede llegar a ser una situación clínica que puede dejar consecuencias en los niños. El objetivo del estudio fue determinarla frecuencia, las características clínicas y epidemiológicas del traumatismo cráneo encefálico leve en el departamento de emergencias pediátricas del hospital de clínicas de San Lorenzo.

\section{MATERIALES Y MÉTODOS}

Estudio observacional descriptivo y retrospectivo. Fueron revisadas las historias clínicas de pacientes con edad comprendida entre 0 a 17 años, que fueron admitidos al departamento de emergencias pediátricas del hospital de Clínicas con diagnóstico de TCE leve, en el periodo comprendido entre noviembre del 2017 a noviembre del 2019. Fuern excluidas las historias clínicas incompletas o mal codificadas, Se consideró TCE leve a todos los pacientes que acudieron a consulta con una puntuación en la escala de glasgow de 15 y la misma puntuación en niños menores de 2 años utilizando la escala de glasgow modificada para lactantes y niños, algunos de ellos consultaron dentro de los 30 minutos de ocurrido el traumatismo (especialmente los lactantes menores) y los mayores luego de los 30 minutos post traumatismo. Se consideraron signos y 
síntomas que presentaron en el tiempo transcurrido luego del traumatismo para evaluar si requerían o no una evaluación clínica más cercana e ingresarlos a la sala de observación.

Se incluyeron en el análisis las variables: edad, sexo, procedencia, mecanismo de traumatismo, altura de la caída, escala de glasgow, hundimiento de cráneo, palidez, otorragia, epistaxis, convulsiones, cefalea, vómitos, requerimiento de TAC de cráneo y hallazgos. La tomografía de cráneo se realizó solo en los pacientes que cumplían con factores de riesgo para lesión cerebral traumática clínicamente importante, (la inconsciencia prolongada (más de un minuto), las alteraciones persistentes del estado mental o las anomalías en el examen neurológico requierieron imágenes urgentes y consulta neurológica o neuroquirúrgica), la radiografía dependía del criterio del médico de guardia en ese momento.
Consideraciones éticas. Se respetó en todo momento los principios éticos para la investigación. No se requirió de consentimiento informado por ser un estudio retrospectivo.

\section{RESULTADOS}

De un total de 65000 consultas generales de noviembre de 2017 hasta diciembre de 2019, 2500 fueron consultas por traumatismos en el Departamento de urgencias pediátricas. El 2,2\% (55/2500) fueron ingresados a sala de observación por traumatismo craneoencefálico leve. Las edades variaron entre 20 días a 17 años (Tabla 1 ).

En cuanto al mecanismo de traumatismo el promedio de altura de las caídas fue de 0,9 0,91 m. Las características se detallan en la Tabla 2.

Tabla 1. Características demográficas y clínicas de los pacientes con traumatismo cráneo encefálico leve en el servicio de emergencias pediátricas del Hospital de Clínicas N=55

\begin{tabular}{lccc}
\hline & & $\mathbf{N}$ & \% \\
\hline SEXO & Masculino & 29 & 52.7 \\
& Femenino & 26 & 47.2 \\
\hline EDAD & $0-28$ dias & 1 & 1.8 \\
& $1-12$ meses & 20 & 36.3 \\
& $1-2$ años & 12 & 21.1 \\
& $3-4$ años & 7 & 12.7 \\
& $4-11$ años & 10 & 18.8 \\
& $12-17$ años & 5 & 9 \\
\hline \multirow{2}{*}{ PROCEDENCIA } & Asunción & 2 & 3.6 \\
& Área Metropolitana & 48 & 87.2 \\
& Interior del País & 5 & 9 \\
\hline
\end{tabular}

Tabla 2. Mecanismo del traumatismo cráneo encefálico leve en el departamento de emergencias pediátricas Hospital de Clínicas N=55

\begin{tabular}{lcc}
\hline CARACTERÍSTICAS & PACIENTES & $\%$ \\
\hline CAÍDA DE ALTURA (= O > A 30cm) & 34 & 61.8 \\
CAÍDA DESDE PROPIA ALTURA & 14 & 25.4 \\
GOLPE CON OBJETO CONTUSO & 4 & 7.2 \\
ACCIDENTE DE TRÁNSITO & 3 & 5.4 \\
\hline
\end{tabular}


En cuanto a la sintomatología se encuentra resumido en la Tabla 3. Todos los pacientes ingresaron al departamento de urgencias vigiles y con un
Glasgow 15/15.91 m. Las características se detallan en la Tabla 2.

Tabla 3. Sintomatología al ingreso de los pacientes con Traumatismo craneoencefálico leve departamento de emergencias pediátricas Hospital de Clínicas N=55

\begin{tabular}{lcc}
\hline SÍNTOMAS & $\mathbf{N}$ & $\mathbf{\%}$ \\
\hline IRRITABILIDAD & 35 & 63.6 \\
VÓMITOS & 27 & 49 \\
PALIDEZ & 13 & 23.3 \\
PÉRDIDA DEL CONOCIMIENTO < A 5 SEG. & 11 & 20 \\
CEFALEA & 8 & 14.5 \\
EPISTAXIS & 4 & 7.2 \\
CONVULSIÓN & 2 & 3.6 \\
OTORREA & 2 & 3.6 \\
HUNDIMIENTO DE CRÁNEO & 2 & 3.6 \\
\hline
\end{tabular}

*1 En la region Frontoparietal y 1 occipital izquierda

Al 54.4\% (30/55) de los pacientes se le practicó una TAC que en el 63\%(19/30) de los pacientes fue normal. En cuanto a los hallazgos radiológicos en pacientes quienes cumplían con criterios de riesgo de lesión intracraneal importante según el médico de guardia que lo recibió se resume en la Tabla 4.

Tabla 4. Hallazgos tomográficos de los pacientes con Traumatismo craneoencefalico leve en el departamento de emergencias pediátricas Hospital de Clinicas $n=55$

\begin{tabular}{lcc}
\hline HALLAZGOS & N & \% \\
\hline HEMATOMA SUBGALEAL & 6 & 20 \\
FRACTURA DE CRÁNEO* & 3 & 5.4 \\
HEMATOMA EPIDURAL OCCIPITAL & 1 & 3.3 \\
CONTUSIÓN CEREBRAL & 1 & 3.3 \\
\hline
\end{tabular}

* 1 = Parietal derecha, 1 temporal derecha y 1 occipital izquierda

\section{DISCUSIÓN}

Actualmente se considera que es un motivo de visita frecuente en países desarrollados como lo menciona Jennet y Guerreiro ${ }^{(5,8)}$ en sus publicaciones, pero no se cuentan con datos precisos de la prevalencia del mismo en infantes a diferencia de lo que sí se puede encontrar en adultos.

En relación al sexo, se ha encontrado un predominio en el sexo masculino, lo cual coincide con varias literaturas, como menciona Powell ${ }^{(9)}$ en sus publicaciones donde encontró que el $65 \%$ de los pacientes fueron del sexo masculino.
La edad con mayor número de casos correspondió al rango etario menor a 2 años, esto puede ser debido al difícil control de los pequeños o descuidos en el cuidado de los padres, seguida por la escolar, adolescentes y neonatos, estos datos coinciden con algunos autores como por ejemplo los aportados por Vos et al. ${ }^{(7)}$ donde más de la mitad de los pacientes con traumatismo craneoencefálico leve son menores de 5 años, otros estudios también mencionan una mayor incidencia en la infancia temprana pero no asi con la etapa de la adolescencia donde también mencionan una mayor incidencia.

En relación a la sintomatología observada en los pacientes, los autores coinciden que la mayoría de 
los pacientes con TCE leve presentan irritabilidad y vómitos, mas aún si se asocian a pérdida de la consciencia y persisten más de una hora del trauma, se vio que en lactantes menores es un signo de alarma de posible lesión traumática importante cerebral por traumatismo por lo que la mayoría de los pacientes que quedaron en observación correspondían a este rango etario (59\%). Además A pesar de la controversia actual existente sobre la exposición a radiación en niños menores de 2 años ${ }^{(20)}$ una gran parte de los mismos fue debido a sus síntomas (anormalidad anatómica en la cabeza al tacto, convulsión, vómitos recurrentes, etc) y también al rango etario menores de dos años, ya que presentan mayor riesgo de lesión intracraneal importante post trauma, la decisión de realizar o no Tomografía de cráneo quedo a cargo del médico de guardia en ese momento, aunque ya existen trabajos como por ejemplo un estudio publicado por Croall et al. ${ }^{(10)}$ donde mencionan que si el único hallazgo es un hematoma del cuero cabelludo, la evidencia de este estudio indica que existe aproximadamente un $0.4 \%$ de riesgo de lesión cerebral traumática clínicamente importante y un $1.7 \%$ de riesgo de lesión cerebral traumática en la $\mathrm{TC}$, que se debería de tener en cuenta a la hora de evaluar y observar a los paciente, especialmente los menores de 2 años de edad antes de someterle a una TC al ingreso. La TAC fue normal en la mayoría de los pacientes sometidos en el presente reporte Solo 1 paciente presento una lesión cerebral traumática clínicamente importante como lo fue un hematoma epidural occipital pero no requirió tratamiento neuroquirúrgico e ingreso a sala de internados para observación y seguimiento. Un estudio realizado por Vos et al. (7) $^{(1)}$ contró que el riesgo de una lesión cerebral traumática clínicamente importante es de aproximadamente
$1 \%$ en pacientes que cumplen criterios de gravedad y solo $0.6 \%$ si el único criterio es un mecanismo de alto riesgo para lesiones, por lo tanto la evidencia de varios estudios más sugiere la observación de pacientes con TCE leve y así disminuir la utilización innecesaria de TC sin perder una lesión cerebral traumática clínicamente importante, para evitar la exposición a radiación en estos niños.

Entre las limitaciones al estudio se encontró la imposibilidad de identificar factores de riesgo para traumatismo craneoencefálico y que en muchos casos el formato de las historias clínicas consultadas no contenía datos más precisos del mecanismo del traumatismo ni tampoco se contó con datos de seguimiento a largo plazo que sería interesante para futuras investigaciones.

\section{CONCLUSIÓN}

La prevalencia de traumatismo craneoencefalico leve en esta muestra de niños fue de 5,5\%. Se observó predominantemente en el sexo masculino y en pacientes que proceden del área metropolitana.

Los menores de 2 años presentaron mayor TCE leve que los mayores a esa edad.

El pronóstico global en base a la escala de glasgow y sintomatologías acompañantes fue mayoritariamente bueno, es decir, con nula mortalidad.

Las manifestaciones clínicas más frecuentes fueron la irritabilidad y los vómitos. La mayoría de los pacientes presentaban una TAC normal.

La indicación de TAC en el TCE leve amerita una mejor evaluación por parte de los médicos de las emergencias pediátricas. 


\section{REFERENCIAS}

1. Practice parameter: the management of concussion in sports (summary statement). Report of the Quality Standards Subcommittee. Neurology. 1997;48(3):581-5. doi: http://dx.doi.org/10.1212/wnl. 48.3 .581

2. Stein SC, Ross SE. The value of computed tomographic scans in patients with low-risk head injuries. Neurosurgery. 1990;26(4):638-40. doi: http://dx.doi.org/10.1097/00006123-199004000-00012

3. Nigrovic LE, Kuppermann N. Children With Minor Blunt Head Trauma Presenting to the Emergency Department. Pediatrics. 2019;144(6):e20191495. doi: http://dx.doi.org/10.1542/peds.2019-1495

4. Jennett B, Frankovyski RF. The epidemiology of head injury. En: Braakman R (Ed). Handbook of Clinical Neurology. Elsevier: New York; 1990. vol 13.

5. Guerriero RM, Proctor MR, Mannix R, Meehan WP 3rd. Epidemiology, trends, assessment and management of sport-related concussion in United States high schools. Curr Opin Pediatr. 2012;24(6):696701. doi: http://dx.doi.org/10.1097/MOP.0b013e328 3595175
6. Centers for Disease Control and Prevention [Internet]. Traumatic Brain Injury \& Concussion. [cited march 27 2017] Available at: http://www.cdc.gov/Tra umaticBrainInjury/index.html

7. Vos PE, Battistin L, Birbamer G, Gerstenbrand F, Potapov A, Prevec T, et al. EFNS guideline on mild traumatic brain injury: report of an EFNS task force. Eur J Neurol. 2002;9(3):207-19. doi: http://dx.doi.org/ 10.1046/j.1468-1331.2002.00407.x

8. Kraus JF, Nourjah P. The epidemiology of mild, uncomplicated brain injury. J Trauma. 1988;28(12): 1637-43. doi: http://dx.doi.org/10.1097/ 00005373198812000-00004

9. Powell JW, Barber-Foss KD. Traumatic brain injury in high school athletes. JAMA. 1999;282(10):958-63. doi: http://dx.doi.org/10.1001/jama.282.10.958

10. Croall ID, Cowie CJ, He J, Peel A, Wood J, Aribisala BS, et al. White matter correlates of cognitive dysfunction after mild traumatic brain injury. Neurology. 2014;83(6):494-501. doi: http://dx.doi.org/10.1212/WNL.0000000000000666 